\title{
Interval Arithmetic in Power Flow Analysis
}

\author{
Zian Wang \\ Student Member \\ Fernando L. Alvarado \\ Senior Member \\ Department of Electrical and Computer Engineering
}

The University of Wisconsin-Madison

\begin{abstract}
The power flow is the fundamental tool for the study of power systems. The data for this problem are subject to uncertainty. This paper uses interval arithmetic to solve the power flow problem. Interval arithmetic takes into consideration the uncertainty of the nodal information, and is able to provide strict bounds for the solutions to the problem: all possible solutions are included within the bounds given by interval arithmetic. Results are compared with those obtainable by Monte Carlo simulations and by the use of stochastic power flows. Object oriented programming techniques make it possible to use interval arithmetic with minimal modifications to existing software. However, to reduce the conservatism inherent in all interval arithmetic computations, the paper describes an iterative method used to obtain the "hull" of the solution set. Keywords: Interval Arithmetic, Power Flow, Non-linear Systems.
\end{abstract}

\section{Introduction}

Power Flow analysis has been one of the most fundamental tools used by power engineers over the decades. The power flow problem is formulated as a set of precisely known nonlinear algebraic equations that must be solved simultaneously. After the solution to these equations is obtained, any system voltage or flow can be determined precisely.

Reality is, of course, different. The models used in power flow analysis are only approximations. The parameter values used in these models are also uncertain. Two types of uncertainty are:

1. Errors in the calculated or measured parameters of the various lines and transformers in the system.

2. Errors in the magnitude of the demand assumed for the system load buses.

Even if parameter uncertainties were not an issue, the power flow problem would be nothing more than a "snapshot" of the system at a given instant. Solutions obtained

Papers presented at the Seventeenth PICA

Conference at the Hyatt Regency Baltimore

Hotel, Baltimore, Maryland, May 7 - 10, 1991

Sponsored by the IEEE Power Engineering Society would be valid only for a single specific system configuration and operating condition. However, the system evolves through time. It appears that it would be more reasonable to ask not what the system looks like at a given instant, but rather to ask for the range of all plausible system conditions that might be encountered as a result of expected uncertainties in demand and other system parameters. Thus, loads and other parameters can be characterized not by a single number but by a range of values, an interval.

Study of a system with interval parameters can be approached by repeated simulations or by interval methods. The repeated simulations approach considers many possible combinations of the parameter values and uses a Monte Carlo approach to the problem, rather than an exhaustive search of extreme values, that would require $2^{K}$ solutions. The approach is similar to the approach often used for production costing studies. Nevertheless, Monte Carlo techniques usually require a large number of cases to attain a reasonable estimate of the variables of interest and their variance.

A practical way to reduce the number of simulations required is to select a limited number of independent load variations. This is often justifiable because loads are often correlated. Selection of load correlations is arbitrary and depends on the experience of engineers [5].

A different approach to the same problem is the use of stochastic techniques for the characterization of uncertainty. Here, rather than assuming that a parameter can be anywhere within a precisely restricted interval, it is assumed that the parameter varies according to some probability distribution. Quite often, the normal distribution is used. Since the early 1970s, many papers have been published on the subject $[1,2,5,6,11,12,13]$. The main advantages of the stochastic power flow model are:

1. All power inputs are probabilistic values.

2. Conventional power flow computations are useable.

3. Branch flows are obtained as probability distribution functions.

Stochastic power flow models have some shortcomings:

1. It is hard to deal with the non-linear relation between the node loads and branch flows.

2. The computational requirements are greater than for conventional power flows. 
This paper describes a new method for taking uncertainty into account during power flow solutions with uncertain input data. The method is based on interval arithmetic. Interval arithmetic is a powerful tool to determine the effects of uncertain data. It can deal with numbers that vary within a range. Interval arithmetic can also consider, in an automatic manner, roundoff error accumulation during numetical computations. The main objective of this paper is to characterize parameter uncertainty in power flow computations by coming up with simple bounds on the solutions that are, in some sense, as small as possible. This paper introduces the basic concepts of interval arithmetic, and then gives a power flow example. Results are compared with those obtainable by Monte Carlo simulations and by the use of stochastic power flows.

\section{Interval Arithmetic}

An interval number $[x, \bar{x}]$ is the set of real numbers $x$ such that $\underline{x} \leq x \leq \bar{x}$. Let $\mathrm{R}$ denote the set of real numbers. Then:

$$
x=[\underline{x}, \bar{x}]:=\{\tilde{x} \in \mathbf{R} \mid \underline{x} \leq \tilde{x} \leq \bar{x}\}
$$

where $\underline{x}, \bar{x}$ are elements of $\mathbf{R}$, with $\underline{x} \leq \bar{x}$, and $\tilde{x}$ is a generic element $\tilde{x} \in x$.

Addition, subtraction, multiplication, and division of intervals are defined as

$$
\begin{aligned}
x+y= & {[\underline{x}+\underline{y}, \bar{x}+\bar{y}] } \\
x-y= & {[\underline{x}-\bar{y}, \bar{x}-\underline{y}] } \\
x * y= & {[\min (\underline{x} * \underline{y}, \underline{x} * \bar{y}, \bar{x} * \underline{y}, \bar{x} * \bar{y}),} \\
& \max (\underline{x} * \underline{y}, \underline{x} * \bar{y}, \bar{x} * \underline{y}, \bar{x} * \bar{y})] \\
x / y= & {[\underline{x}, \bar{x}] *[1 / \bar{y}, 1 / \underline{y}] \quad \text { if } 0 \notin[\underline{y}, \bar{y}] }
\end{aligned}
$$

Division is not defined if $0 \in[y, \bar{y}]$.

Only some of the algebraic laws valid for real numbers remain valid for intervals; other laws only hold in a weaker form. Interval addition and multiplication are associative and commutative:

\section{Commutativity:}

$$
a+b=b+a \quad a b=b a
$$

Associativity:

$$
(a+b) \pm c=a+(b \pm c) \quad(a b) c=a(b c)
$$

Neutral element:

$$
a+0=0+a=a \quad 1 * a=a * 1=a
$$

However, the distributive law does not always hold for interval arithmetic. For instance, $a+x=b$ does not imply $x=b-a$. As an example,

$$
[1,2]+[3,4]=[4,6]
$$

but

$$
[4,6]-[1,2]=[2,5]
$$

An important property, referred to as subdistributivity, does hold. It is given by the set inclusion relationship:

$$
a b \pm a c \supseteq a *(b \pm c), \quad a c \pm b c \supseteq(a \pm b) * c
$$

and a subcancellation law also holds

$$
\begin{aligned}
(a+c)-(b+c) \supseteq a-b, & (a b / b c) & \supseteq a / b \\
0 \in a-a, & 1 & \in a / a
\end{aligned}
$$

Let IR denote the set of real intervals. Define an interval as thin if $\underline{x}=\bar{x}$. All real numbers $x \in \mathbf{R}$ can be represented as thin intervals.

The failure of the distributive law often causes overestimation. Nevertheless, the subdistributive law in IR, the weak substitute for the distributive law in $R$, is a useful tool. In some special cases, the distributive law remains valid. Let $a, b, c, \in \mathbf{I R}$, then

$$
a *(b \pm c)=a b \pm a c \quad \text { if } a \text { is thin }
$$$$
a *(b+c)=a b+a c \text { if } b, c \geq 0 \text { or } b, c \leq 0
$$

$a *(b-c)=a b-a c$ if $b \geq 0 \geq c$ or $b \leq 0 \leq c$

Power flow problems require non-linear equations. Three popular iteration operators for the solution of interval nonlinear equations are the Newton operator [14], the Krawczyk operator [10], and the Hansen-Sengupta operator [8]. The most popular one is the Newton operator:

$$
N(x, \check{x}):=\check{x}-F^{\prime}(x)^{-1} F(\check{x})
$$

where $\dot{x}$ is the midpoint of the interval $[\underline{x}, \bar{x}]$, defined as:

$$
\check{x}:=(\bar{x}+\underline{x}) / 2
$$

$F^{\prime}(x)$ is the interval Jacobian matrix. For each iteration, we need to solve interval linear equations:

$$
F^{\prime}(x) \Delta x=F(\check{x}) \quad \text { for } \quad \Delta x
$$

Thus, solution of interval non-linear equations reduces (like in the ordinary case) to the solutions of linear equation, but using interval arithmetic. However, the solution of interval linear equations (which is at the heart of the nonlinear iterative solution) is a very different proposition from the solution of ordinary linear equations. For one, the exact solution set of these equations cannot be characterized by an interval vector. The solution set has a very complex non-convex structure. Figure 1 illustrates the solution set for a two dimensional interval equations. The black region in figure 1 is the exact solution set. The solution set cannot be characterized as an interval. It is possible, however, to find the hull of the solution set, where the hull is defined as the smallest interval vector that contains the solution set. The hull of the solution is also illustrated in figure 1 , where the hull of the solution set is: $x=\left(\begin{array}{l}x_{1} \\ x_{2}\end{array}\right)=\left(\begin{array}{l}{[-4,4]} \\ {[-4,4]}\end{array}\right)$. The hull is indicated by the shaded region. It contains, in addition to the entire solution set, many non-solutions.

The second major difference is that LDU decomposition to solve interval linear equations does not usually give the hull of the solution set. The hull can be found by either explicit inverse of matrices or by iterative methods.

Solving interval linear equations means to obtain the hull of the solution set. There are several methods to solve interval linear equations. These include:

1. Krawczyk's method [10].

2. Interval Gauss-Seidel iteration [15].

3. $L D U$ Decomposition. 


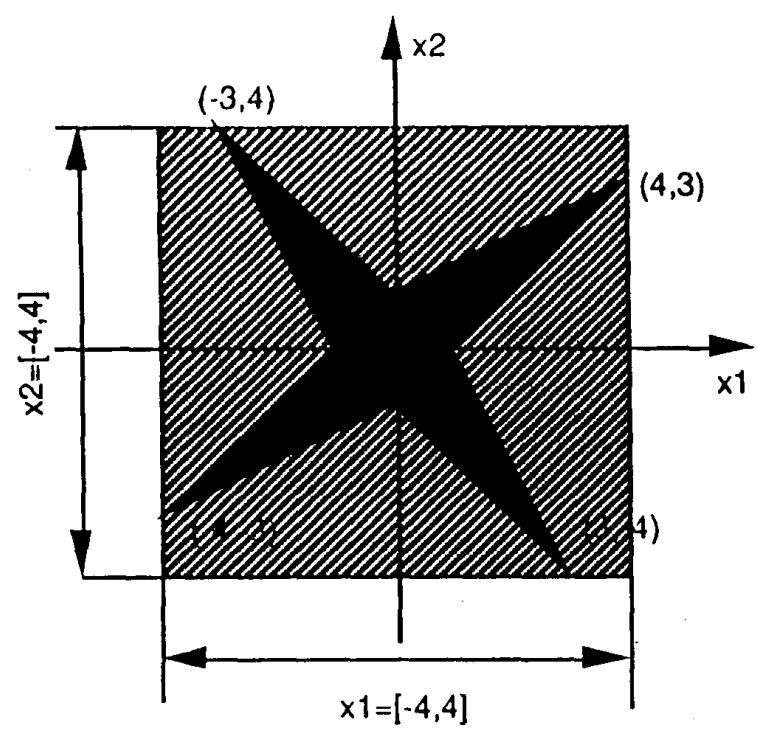

Fig. 1: Solution of 2 by 2 interval equations

LDU decomposition is not the best method to solve linear equations because of excessive interval growth. However, implementation of interval arithmetic using $L D U$ decomposition, if this is desired, can be accomplished with little modification to current software for ordinary power flows, which in most cases uses ordered LDU factorization. The implementation is simple: every operation performed by the computer is replaced by an interval operation. This can be accomplished in a global manner if object-oriented techniques are used: data is defined as an object, then a new type of object (an "interval" object) is defined, and new arithmetic rules are substituted. While implementation following these ideas becomes quite simple, the results obtained are normally far too conservative to be of practical value. The use of better ordering techniques can reduce interval growth, but a basic problem remains. The best solution to the problem of excessive conservatism in linear equation solving is to abandon direct methods and use either Krawczyk's method or interval Gauss Seidel iterations.

The most widely used method to solve interval linear equations is Gauss-Seidel iteration. While this may seem like a bad idea, the purpose of Gauss-Seidel iterations here is not to solve the power flow problem, but to solve the linear equations that result from Newton's method, and to do so with as little conservatism as possible. In order to converge to a solution, this method requires that the matrix be an M-matrix. M-matrices are defined as:

Definition $1 A$ matrix $A$ is an M-matrix if $A_{i k} \leq 0$ for all $i \neq k$, and $A u>0$ for some positive vector $u \in \mathbf{I R}^{\mathbf{n}}$.

If the matrix is an M-matrix, the hull of the solution set can be guaranteed by the Gauss-Seidel method; if the matrix is not an M-matrix, the solution may become infinite. For general matrices which are not M-matrices, preconditioning has to be used. Preconditioning [9] means multiplication of both sides of the equation by a preconditioning matrix. Using preconditioning, some matrices which are not $\mathrm{M}$-matrices may become M-matrices.

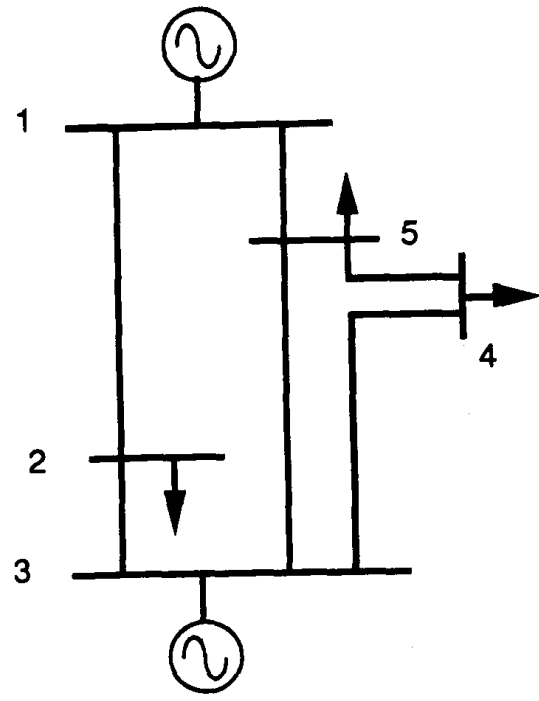

Fig. 2: A 5-bus power system

To summarize, solving the power flow problem using interval arithmetic can be done by first linearizing the problem (as in the ordinary arithmetic power flow case). However, the resulting linear equations must be solved by a GaussSeidel iterative process instead of by direct LDU factorization. The solution obtained is conservative in that it contains all solution points, but may contain non-solutions as well. This limitation is not an algorithmic limitation, but rather a fundamental limitations of attempting to express solutions as simple intervals when in reality they are not.

\section{Power Flow Application: An Example}

The standard interval power flow equations can be expressed as:

$$
\begin{aligned}
& \mathbf{y}=f(\mathbf{x}) \\
& \mathbf{z}=g(\mathbf{x})
\end{aligned}
$$

where $f$ and $g$ are functions and $\mathbf{y}, \mathbf{x}$ and $\mathbf{z}$ are interval vectors:

$\mathbf{y}=$ real and reactive power injections (inputs)

$\mathbf{x}=$ voltage magnitudes and angles (states)

$\mathrm{z}=$ line power flows (outputs)

A 5-bus example from [3] is shown in figure 2. The system is assumed to operate at normal conditions but all power demands may vary within certain ranges rather than have precise values. The problem is to find the interval values of the power flow.

This section compares three different approaches to the characterization of uncertainty in power systems: stochastic power flows, interval arithmetic methods and Monte Carlo simulation. The objective is to characterize the uncertainty in line flows.

Each method requires slightly different assumptions. The basic assumption is that demands at buses are uncertain over a given interval. In the interval power flow, these intervals can be used directly. The line flows obtained will, 
however, be conservative. In the stochastic power flow approach, the intervals must be characterized as a probability distribution. If the intervals are treated as a uniform probability distribution, the mean and variance of the distribution can be calculated, and the uniform distribution can be replaced by a normal distribution of the same mean and variance. This means that the results will be slightly different, in that it is possible for the solution to have some values outside those predicted by the more strict bounds from interval arithmetic. Finally, the Monte Carlo approach requires an assumption as to the probability distribution of demand values within the interval. A uniform probability density function over the entire interval is assumed. Thus, Monte Carlo simulations should always be within the predictions of interval methods.

\subsection{Stochastic Power Flows}

There are several stochastic power flow methods: Simple Algorithms [1, 5, 6], Multilinearization Algorithms [2], Correlation between Inputs $[11,13]$. This paper compares results using the algorithm of $[6]$, but similar results are expected from any of the others. Table 1 gives the input vector $y$, the injected real and reactive power interval for all the buses. In order to use stochastic power flows, a normal probability distribution is needed. First, assume that the intervals in Table 1 characterize uniform probability density functions. The mean value and the variance for these can be computed from [7]:

$$
\begin{aligned}
\check{y} & =\frac{1}{2}(\underline{y}+\bar{y}) \\
\sigma_{y}^{2} & =\frac{1}{12}(\bar{y}-\underline{y})^{2}
\end{aligned}
$$

Using the mean values of y (i.e, real and reactive power injection), the mean values of the state variables can be computed using conventional power flow methods. After this is done, the variances of state variables and branch load flows can be calculated using the formulas from [6]:

$$
\begin{gathered}
\sigma_{x}^{2}=\operatorname{diag}\left(J^{t} V^{-1} J\right)^{-1} \\
\sigma_{z}^{2}=\operatorname{diag}\left(K\left(J^{t} V^{-1} J\right)^{-1} K^{t}\right)
\end{gathered}
$$

where $J$ is the Jacobian of the load flow equations, $V$ is a diagonal matrix of variances of injected power, and $K$ is the first order matrix from the Taylor series expansion of $g(\mathbf{x})$.

To perform a meaningful comparison against interval methods, the solution results are characterized as an interval of $\pm 3 \sigma$ around the mean. That is:

$$
\begin{array}{ll}
\underline{x}=\check{x}-3 \sigma_{x}, & \bar{x}=\check{x}+3 \sigma_{x} \\
\underline{z}=\dot{z}-3 \sigma_{z}, & \bar{z}=\check{z}+3 \sigma_{z}
\end{array}
$$

Numerical results are shown in the Table 2 for all variables. Figure 3 illustrates the resulting assumed normal probability distribution computed for one of the lines, line 2-1. Figure 4 illustrates the same results as Table 2. This figure shows that the conservatism introduced by interval methods on the state variables is modest. However, the conservatism on derived variables (flows, which depend on differences of state variables) is much greater.

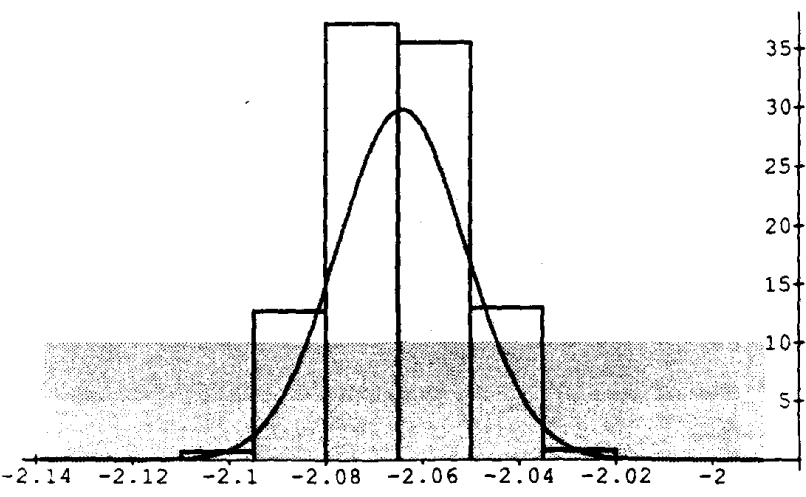

Fig. 3: Comparison of $P_{21}$ flow. Bell-shaped curve is from stochastic power flows, step-wise distribution is obtained from 1000 Monte-Carlo simulations, and shaded area is the hull of the solution using interval methods.

Table 1: Base Values of the 5-bus Power System

\begin{tabular}{|c|c|c|c|c|}
\hline Bus & Type & $P$ & $Q$ & $V$ \\
\hline 1 & ref & & & 1.040 \\
2 & load & {$[1.15,1.2]$} & {$[0.6,0.65]$} & \\
3 & gen & {$[1.15,1.2]$} & & 1.020 \\
4 & load & {$[0.8,0.85]$} & {$[0.3,0.35]$} & \\
5 & load & {$[1.0,1.05]$} & {$[0.4,0.45]$} & \\
\hline
\end{tabular}

\subsection{Interval Method}

This subsection uses interval arithmetic to solve this same power flow problem with interval input data. To solve interval non-linear equations, the Newton operator is used. For each iteration, the Gauss-Seidel Method is used to solve the interval linear equations. Experience using a variety of iteration schemes with several linear solvers suggests that the ideal combination of methods is the Newton operator for the non-linear interactions and the Gauss-Seidel iteration procedure for the required interval linear solver. Table 2 shows the results of interval state variables and interval power values. If the range of interval values is too large (for example, $\left.P_{3}=[1.0,2.0]\right)$, the results do not converge. The interval Jacobian matrix cannot be converted into an $M$ matrix even after preconditioning. The Appendix contains details of the interval iterative process for two simple well behaved examples.

\subsection{Monte Carlo}

The third method used to validate the power flow results with uncertain data is Monte Carlo simulation. For this simulation, 1000 different values of power injections within the intervals given in Table 1 were selected and conventional power flow simulations were performed. Interval solutions were obtained by monitoring the largest and the smallest values of line flows obtained during all 1000 simulations. Table 2 shows that all the values are within the range predicted by the interval method. 


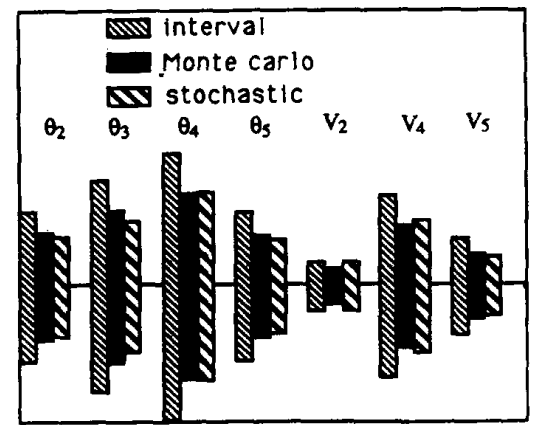

(a) State Variables.

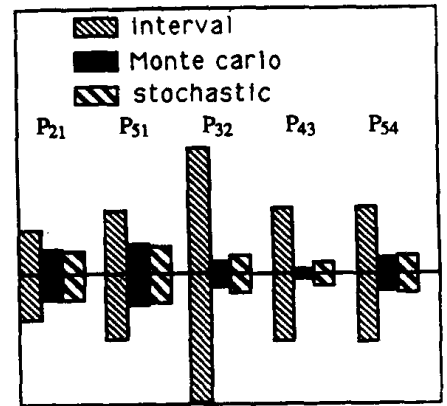

(b) Active Power Flows.

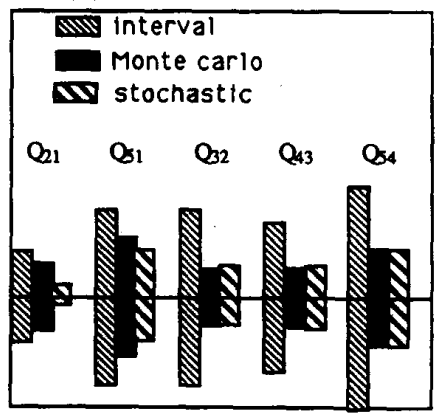

(c) Reactive Power Flows.

Fig. 4: Illustration of relative uncertainty intervals. All intervals within each figure use the same vertical scale. The centers of all intervals have been aligned.

\subsection{Comparison of Methods}

Since the methods differ in some fundamental ways, an exact direct comparison of computational requirements is not possible. Each method has some strengths and some limitations. In our own experiments, the implementations were rather different in nature. The interval arithmetic implementation was done using an object oriented version of the Sparse Matrix Manipulation System [4], the Monte Carlo approach used a conventional power flow, and the Stochastic Power Flow was done with some help from MatLab. However, some features did emerge. We were able to collect statistics on numbers of multiplications. Based on these, the Monte Carlo approach is hundreds of times slower than the other two methods. The stochastic power flow was
Table 2: Comparison of Solutions using Interval Methods, Monte Carlo Simulations, and Stochastic Power Flows

\begin{tabular}{|c|c|c|c|}
\hline & $\begin{array}{c}\text { Interval } \\
\text { Method }\end{array}$ & $\begin{array}{c}\text { Monte Carlo } \\
\text { Simulation }\end{array}$ & $\begin{array}{c}\text { Stochastic } \\
\text { Power Flow }\end{array}$ \\
\hline \hline$\theta_{2}$ & {$[-0.1346,-0.1113]$} & {$[-0.1313,-0.1147]$} & {$[-0.1306,-0.1152]$} \\
\hline$\theta_{3}$ & {$[-0.1011,-0.0685]$} & {$[-0.0967,-0.0731]$} & {$[-0.0949,-0.0746]$} \\
\hline$\theta_{4}$ & {$[-0.2559,-0.2149]$} & {$[-0.2499,-0.2213]$} & {$[-0.2498,-0.2210]$} \\
\hline$\theta_{5}$ & {$[-0.1444,-0.1216]$} & {$[-0.1408,-0.1250]$} & {$[-0.1402,-0.1257]$} \\
\hline$V_{2}$ & {$[0.9519,0.9595]$} & {$[0.9527,0.9586]$} & {$[0.9520,0.9594]$} \\
\hline$V_{4}$ & {$[0.8711,0.8990]$} & {$[0.8755,0.8943]$} & {$[0.8748,0.8953]$} \\
\hline$V_{5}$ & {$[0.9413,0.9561]$} & {$[0.9436,0.9536]$} & {$[0.9442,0.9532]$} \\
\hline$P_{21}$ & {$[-2.1391,-1.9898]$} & {$[-2.1027,-2.0177]$} & {$[-2.1044,-2.0242]$} \\
\hline$Q_{21}$ & {$[-5.4004,-5.3099]$} & {$[-5.3887,-5.3211]$} & {$[-5.3655,-5.3452]$} \\
\hline$P_{51}$ & {$[-2.8881,-2.6722]$} & {$[-2.8199,-2.7183]$} & {$[-2.8276,-2.7317]$} \\
\hline$Q_{51}$ & {$[-7.1644,-6.9907]$} & {$[-7.1359,-7.0180]$} & {$[-7.1226,-7.0329]$} \\
\hline$P_{32}$ & {$[-1.7276,-1.3038]$} & {$[-1.5366,-1.4946]$} & {$[-1.5472,-1.4837]$} \\
\hline$Q_{32}$ & {$[-7.4424,-7.2683]$} & {$[-7.3856,-7.3296]$} & {$[-7.3877,-7.3283]$} \\
\hline$P_{43}$ & {$[-1.1166,-0.8938]$} & {$[-1.0089,-0.9924]$} & {$[-1.0232,-0.9855]$} \\
\hline$Q_{43}$ & {$[-2.4791,-2.3294]$} & {$[-2.4350,-2.3768]$} & {$[-2.4363,-2.3742]$} \\
\hline$P_{54}$ & {$[-0.5744,-0.3485]$} & {$[-0.4874,-0.4308]$} & {$[-0.4904,-0.4281]$} \\
\hline$Q_{54}$ & {$[-3.3098,-3.0889]$} & {$[-3.2469,-3.1514]$} & {$[-3.2479,-3.1516]$} \\
\hline
\end{tabular}

comparable to the interval arithmetic method. The interval arithmetic method did not take particular advantage of many known computational shortcuts available to interval computations. Convergence to the hull was extremely rapid (one or two iterations).

Another relevant point of comparison is how the methods behave as the size of the intervals increase. Figure 5 illustrates the behavior of the ratio in the uncertainty interval for the methods. It illustrates that, as the uncertainty intervals grow, the behavior of both stochastic power flows and Monte-Carlo methods stays constant. However, the degree of conservatism introduced by interval methods worsens as the interval sizes increase.

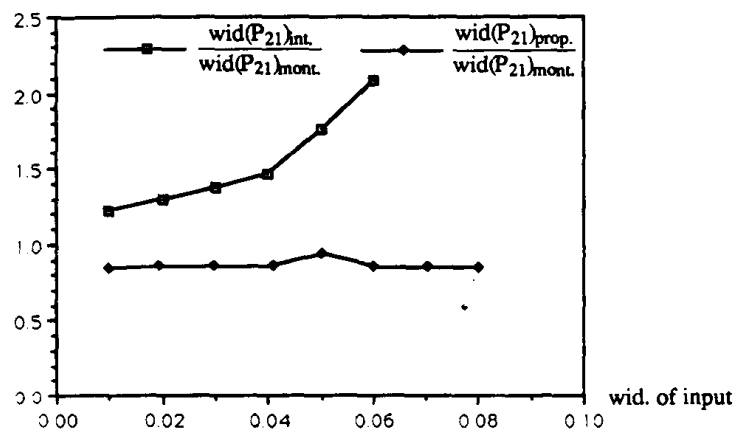

Fig. 5: Ratio of interval widths as a function of uncertainty in the input. The conservatism introduced by interval methods worsens as data uncertainty increases.

We have not yet performed extensive tests of specific comparative power flow studies in these larger systems. The computational requirements per iteration for interval methods scale with system size in the same manner as ordinary "first neighbor" sparse matrix methods do, with the advan- 
Table 3: Multiplications vs. system size for the solution of linear equations. Top row is number of multiplications required for interval solution, assuming 4 iterations to refine the hull. Bottom row are multiplications using ordinary LDU factorization (scheme 2 ordering).

\begin{tabular}{|r|r|r|r|r|r|}
\hline Size & 118 & 352 & 707 & 1084 & 1993 \\
\hline Interval multiplications & 7616 & 19168 & 42320 & 66384 & 138384 \\
\hline Ordinary LDU solution & 1178 & 2788 & $\mathbf{8 3 5 1}$ & 11626 & 26293 \\
\hline
\end{tabular}

tage that fill-in does not play a role. Extra arithmetic is required for every interval elementary operation, but this represents a constant factor. Also, an added burden is due to the preconditioning of the matrix, particularly as the intervals become larger. The number of iterations is more a function of how wide the intervals are, which is related to whether the matrix is an M-matrix. For small intervals (the usual case), convergence is not an issue even in large systems. As the intervals become large, the Jacobian ceases to be an M-matrix and convergence suffers. Further experimentation is needed to determine the exact properties of these matrices in large systems.

In place of tests on larger power flow cases, we offer comparative tests on the growth in the number of multiplications per iteration when solving well conditioned large interval linear problems. Table 3 illustrates the number of multiplications required to perform a single linear solve using interval arithmetic assuming that four iterations are sufficient. The interval solution multiplications are compared with the solution of linear equations with the same topology using LDU factorization and ordinary arithmetic. The assumption of four iterations to solve a linear interval problem may not always be valid. At this time we lack sufficent experience to generalize this result. However, preliminary indications are that in many cases it is sufficient. Because these linear cases are assumed to be well behaved, pre-conditioning overhead has not been considered.

\section{Conclusions}

Interval method can deal with uncertain input data in power flow problems. If input data vary within relatively small ranges, good results that contain all possible solutions are obtained. The proposed method has been validated against Monte Carlo simulations and stochastic power flow results. Interval methods have proven computationally superior to Monte Carlo simulations and in some cases comparable to Stochastic Power Flows, although somewhat more conservative. The ability of interval methods to automatically consider numerical roundoff is a welcome side benefit of interval methods. Interval methods are compatible with sparsity preservation provided algorithms are chosen with care. Although interval methods cannot be recommended for general use at this time, they offer enough intriguing features to warrant further consideration.

\section{Acknowledgement}

This work was supported by NSF under contracts ECS8822654 and ECS-8907391.

\section{REFERENCES}

[1] Allan, R. N., A. M. Leite da Silva, and R. C. Burchett, "Evaluation Methods and Accuracy in Probabilistic Load Flow Solutions," IEEE Trans. on PAS, Vol. PAS-100, No. 5, pp. 2539-2546, May 1981.

[2] Allan, R. N., and A. M. Leite da Silva, "Probabilistic Load Flow Using Multilinearizations," IEE Proc., Vol. 128, Pt. C, No. 5, pp. 280-287, Sept. 1981.

[3] Alvarado, F. L., and T. H. Jung, "Direct Detection of Voltage Collapse Conditions," EPRI Report EL-6183, pp. 5.235.38, Jan. 1989.

[4] Alvarado, F. L., "Manipulation and Visualization of Sparse Matrices," ORSA Journal on Computing, Vol. 2, No. 2, pp. 186-207, Spring 1990.

[5] Borkowska, B., "Probabilistic Load Flow," IEEE Trans. on PAS, Vol. PAS-93, No. 3, pp. 752-755, May/June 1974.

[6] Dopazo, J. F., O. A. Klitin, and A. M. Sasson, "Stochastic Load Flow," IEEE Trans. on PAS, Vol. PAS-94, No. 2, pp. 299-309, March/Apr. 1975.

[7] Cooper, G. R., and C. D. McGillem, Probabilistic Methods of Signal and System Analysis, Holt, Rinehart and Winston, Inc. 1971.

[8] Hansen, E. and S. Sengupta, "Bounding Solutions of Systems of Equations Using Interval Analysis," BIT Vol. 21, pp. 203-211 1981.

[9] Hansen, E. and R. Smith, "Interval Arithmetic in Matrix Computations, Part II," SIAM Journal of Numerical Analysis, 4, 1967, pp. 1-9.

[10] Krawczyk, R., "Newton-Algorithmen zur Bestimmung von Nullstellen mit Fehlerschranken," Computing Vol. 4, 1969, pp. 187-201.

[11] Leite da Silva, A. M., V. L. Arienti, and R. N. Allan, "Probabilistic Load Flow Considering Dependence Between Input Nodal Powers," IEEE Trans. on PAS, Vol. PAS-103, No. 6, pp. 1524-1530, June 1984.

[12] Leite da Silva, A. M., "Probabilistic Techniques in Load Flow Problems," IEEE International Symposium on Circuits and Systems, Vol. 1, pp. 381-384, 1984.

[13] Meliopoulos, S., G. J. Cokkinides and X. Y. Chao, "A New Probabilistic Power Flow Analysis Method," IEEE Transactions on Power Systems, Vol. 5, No. 1, pp. 182-190, Feb. 1990.

[14] Moore, R. E., "Interval Analysis." Prentice-Hall, Englewood Cliffs, N. J. 1966.

[15] Ris, F. N., "Interval Analysis and Applications to Linear Algebra," D. Phil. Thesis, Oxford, 1972.

\section{Appendix: Simple Interval Equations}

This appendix describes by means of two simple examples some of the details of interval solutions. Consider first the solution of the equation illustrated in figure 6 :

$$
y=x^{2}+[1,3] x-[7,9]=0
$$

We seek a solution in the interval $x^{0}=[0,6]$. This interval is illustrated as interval a.

The symbolic interval Jacobian (a scalar, in this case) is:

$$
J=\frac{d y}{d x}=2 x+[1,3]
$$

Select a specific point $\tilde{\boldsymbol{x}} \in \boldsymbol{x}$ within the interval:

$$
\tilde{x}=\operatorname{mid}(x)=[3,3]
$$




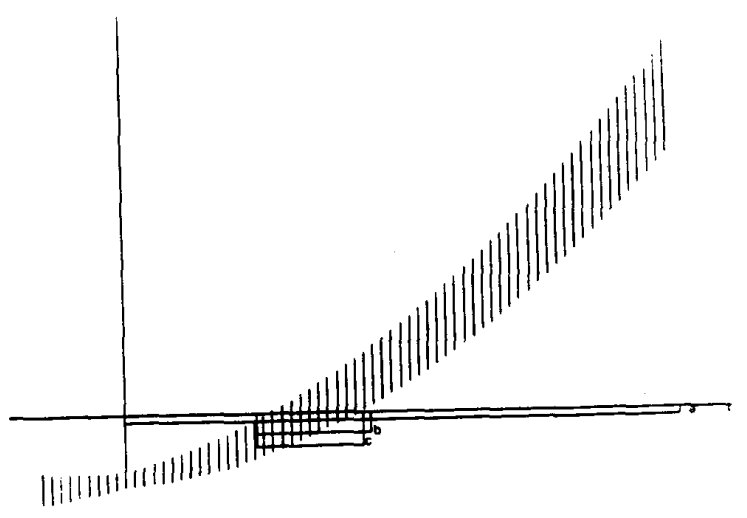

Fig. 6: A scalar nonlinear interval equaliun.

Evaluate the function $y$ at this point $\tilde{x}$ :

$$
\begin{aligned}
y(\tilde{x}) & =([3,3])^{2}+[1,3] \cdot[3,3]-[7,9] \\
& =[3,11]
\end{aligned}
$$

Evaluate the Jacobian:

$$
J(\tilde{x})=[7,9]
$$

Solve the interval linear equation:

$$
[7,9] \Delta x=[3,11]
$$

Because we are dealing with a single equation, simple interval division yields the hull of the solution (which in this case it is the entire solution set):

$$
\Delta x=[0.333,1.571]
$$

Interval subtraction of $\Delta x$ from $\tilde{x}$ and intersection with the initial guess interval $x$ yields the new guess:

$$
\begin{aligned}
x^{1} & =(\tilde{x}-\Delta x) \cap x^{0} \\
& =[1.429,2.667]
\end{aligned}
$$

This interval is illustrated as $b$ in figure 6 .

We determine a new mid-point $([2.048,2.048])$ and repeat the computation. Our next interval is:

$$
x^{2}=[1.428,2.589]
$$

which is illustrated in as interval $c$ in figure 6 .

The second example illustrates a set of equations. Let the interval equations be:

$$
\begin{array}{r}
\epsilon_{1}=x^{2}+y^{2}-[3,5]=0 \\
\epsilon_{2}=x^{2}+[8,10] y^{2}-9=0
\end{array}
$$

These equations are illustrated in figure 7 . The symbolic interval jacobian for these equations is:

$$
J=\left[\begin{array}{cc}
{[2,2] x} & {[2,2] y} \\
{[2,2] x} & {[16,20] y}
\end{array}\right]
$$

Assume an initial guess $x^{0}=[[0.5,3],[0.4,2]]^{t}$, illustrated as region a in figure 7 . Using the mid-point $[\tilde{x}, \tilde{y}]$ for this region, we obtain the linear equations:

$$
\left[J\left(x^{0}, y^{0}\right)\right]\left[\begin{array}{c}
\Delta x \\
\Delta y
\end{array}\right]=\left[\begin{array}{l}
\epsilon_{1} \\
\epsilon_{2}
\end{array}\right]
$$

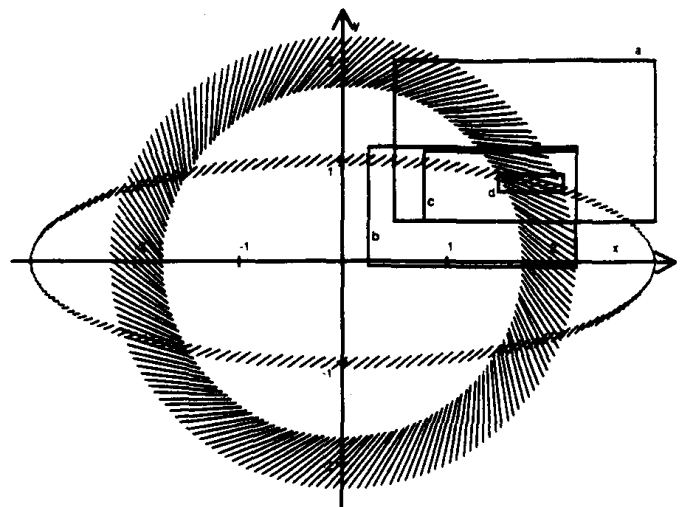

Iig. 7: Solution of two interval nonlinear equations by Newton's method. The linear equations are solved by interval Gauss-Seidel.

These linear interval equations are solved by interval Gauss-Seidel iteration. The result converges to a solution. When this solution is subtracted from the initial mid-point vector $[\tilde{x}, \tilde{y}]$, the result is:

$$
\left[\begin{array}{l}
x^{1} \\
y^{1}
\end{array}\right]=\left[\begin{array}{c}
{[0.247,2.247]} \\
{[-0.44,1.136]}
\end{array}\right]
$$

This vector is illustrated as region $b$ in figure 7. A portion of this region is outside the assumed region. Intersect this region with the original one and proceed. The GaussSeidel iterations to solve the linear equations continue until convergence ( 3 iterations). Convergence yields:

$$
\left[\begin{array}{l}
x^{1} \\
y^{1}
\end{array}\right]=\left[\begin{array}{l}
{[0.787,2.247]} \\
{[0.400,1.095]}
\end{array}\right]
$$

This region is illustrated as $c$ in figure 7. The process is repeated by re-linearizing the equations and re-solving. After 3 additional Newton steps:

$$
\left[\begin{array}{l}
x^{4} \\
y^{4}
\end{array}\right]=\left[\begin{array}{l}
{[1.500,2.128]} \\
{[0.701,0.866]}
\end{array}\right]
$$

This region is illustrated as d, and corresponds to the hull of the solution.

Zian Wang was born in Shanghai, China in 1957. She recieved her B. S. and M. S. degrees from Xian Jiaotong University, China, in 1982 and 1984 respectively. She is currently pursuing her $\mathrm{Ph}$. $\mathrm{D}$. degree at the University of Wisconsin - Madison. Her research interests are interval arithmetic, sparse matrix technology and their application to power systems.

Fernando L. Alvarado obtained his BS degree from the National University of Engineering in Lima, Peru, in 1967, his MS degree from Clarkson University in Potsdam, New York, in 1969 and his Ph. D. degree from the University of Michigan in Ann Arbor Michigan in 1972. He is currently a Professor at the University of Wisconsin in Madison, Wisconsin, in the department of Electrical and Computer Engineering. His main interests areas are computer applications to power systems, large scale program, sparse matrices, software concepts and power system security and economics. 


\section{Discussion}

A. P. Sakis Meliopoulos and F. Xia: The authors should be commended for introducing interval arithmetic in power flow analysis which can take into consideration the uncertainty of the bus injections and provide the range of the solution vector. The concept of the proposed method is excellent. Many times there is uncertainty in the input data which can not be quantified with a specific distribution function. In these cases representing the uncertainty with an interval is a practical approach. The results of this approach can be characterized as 'negative' in the sense that interval arithmetic exaggerates the uncertainty. However the value of the paper is high because it delineates the limitations of interval arithmetic.

The application of interval arithmetic to the power flow problem as proposed in the paper assumes total independence among the power injections. This is not true in real life. We believe that a better model will be to assume some kind of correlation among the power injections. One suggested approach is to model the power injections with a small number of variables as follows:

$$
P=P_{0}+P_{1} v_{1}+P_{2} v_{2}+\ldots+P_{m} v_{m}
$$

where

$P$ is the vector of uncertainty in power injections

$v$ are a set of variables

$m$ is the number of variables $v$ (small)

This model will be a variation of the model proposed in [1]. The variables $v$ can be assumed to be independent variables representing the uncertainty interval. The gained advantage of this model is that now only a small number of variables are to be dealt with.

In dealing with uncertainty in power flow solutions one seek to compute the distribution of some output quantities (i.e. circuit flow, bus voltage magnitude, etc). It appears to us that the authors in their application of interval arithmetic, first compute the 'bull' of the solution set (state vector) and then proceed to compute the uncertainty of output quantities. What will be the computational ramifications and the quality of the results if first a linearized expression of output quantities is developed and subsequently interval arithmetic is applied to this expression? We would expect that this approach will provide better results because the uncertainty will not be exaggerated as much as in the proposed approach. Coupled with an efficient linearization procedure the approach may be also practical.

Another question is as follows. Assume that the uncertainty is represented with a uniform distribution over an interval. Then the computational procedure may be identical to the one proposed in the paper with the exception that the interval arithmetic is replaced with convolutions. One can argue that the results of this approach will be realistic but the computational requirements may be higher than those of interval arithmetic. Can the authors comment on the comparative merits of these two approaches?

\section{Reference}

[1] A. P. Meliopoulos, G. J. Cokkinides, and X. Y. Chao, "A New Probabilistic Power Analysis Method," IEEE Transactions on Power Systems, Vol. PWRS-5, No. 1, February 1990.

Manuscript received May 16, 1991.

Vladimiro Miranda (Inesc, Portugal): The authors describe a new method for taking uncertainty into account during power flow solutions with uncertain input data, based on interval arithmetic.

However, interval operations can be seen as only a special case of fuzzy set operations, each interval being represented by a membership function of constant value 1 between its bounds and 0 outside.

If one has complete knowledge on some piece of evidence, then the right numeric representation is a certain or deterministic number. If the knowledge is not complete, but one knows that there is a frame of repetition of events, with fixed laws (though not explicit) governing it, one can and should represent information by random members, which come associated with probability distributions. However, if these conditions are not met, but one still has qualitative (or other) information on the phenomena to be represented, fuzzy set representation should be used-or fuzzy numbers, which come associated with possibility distributions

Fuzzy set modelling of power system data where uncertainties are not of probabilistic type has been done and published since 1989. A list of references is included at the end of this discussion. Models vary from fuzzy load curve modelling to fuzzy power flow analysis, fuzzy dispatch, fuzzy risk analysis, fuzzy reliability studies and fuzzy optimization.

Therefore, we feel that a different focus should have been given to the paper. Interval arithmetic is not the adequate way of modelling uncertainties, but certainly is a necessary tool to deal with fuzzy number operations, when parameterized with $\alpha$-cut concepts.

We disagree with comparisons of results to any stochastic model, for the following reasons:

a) A new technique should be carefully explained to people that are not used to it.

b) People used to probabilistic methods tend to think at first that someone is trying to replace their well known and faithful tool by another thing.

c) Results are not comparable because the underlying assumptions on the information one has are very different-one is quantitative, though random, the other is qualitative.

d) Probabilistic and possibilistic are not competing but cooperative approaches, and both should be used in Power System modelling.

These are some of the reasons why we feel that this paper does not have its pedagogic value as high as it could have. In any case, the authors should be congratulated for addressing the problem of non probabilistic uncertainties representation, and encouraged in their interval arithmetic application research, in order to provide tools for the manipulation of power system models based on the fuzzy set theory.

\section{References}

[1] V. Miranda M Matos "A Holistic Approach in Multi Criteria Decision Aid," in Improving Decision Making in Organizations, A. G. Lockett Ed., Lecture Notes in Economics and Mathematical Sciences, Springer Verlag, 1989.

[2] V. Miranda, M. Matos, "Distribution System Planning with Fuzzy Models and Techniques," Proceedings of CIRED 89 (IEE), Brighton, U.K., May 1989.

[3] V. Miranda, "Cálculos de fiabilidade em sistemas de energia com indices imprecisos" (in portuguese), Proceedings of I Jornadas Hispano-Lusas en Ingeneria Electrica, Vigo, Spain, July 1990.

[4] V. Miranda, M. Matos, J. T. Saraiva, "Fuzzy Load Flow-New Algorithms Incorporating Uncertain Generation and Load Representation", Proceedings of the 10th PSCC, Graz, Austria, August 1990, Ed. Butterworths, London.

[5] V. Miranda, "Using Fuzzy Reliability Indices in a Decision Aid Environment for Establishing Interconnection and Switching Location Policies," Proceedings of CIRED 91 (IEE), Liége, Belgium, April.

[6] V. Miranda, J. T. Saraiva, "Fuzzy Modelling of Power System Optimal Power Flow", Proceedings of IEEE PICA Conference, Baltimore, USA, May 1991.

Manuscript received June 3, 1991.
F. ALVARADO and Z. WAIJG (The University of Wisconsin, Madison, WI): We thank the discussers for their valuable comments. In response to tie Dr. Meliopoulos and Mr. Xia, we agree that it is indesd appropriate to assume that demand uncertainties are correlated variables, perhaps with as little as a single degree of freedom [C1]. If this is done and if in addition only uncertainties associated with injections are considered, it becomes possible to "defer" the use of interval methods until after analytic expressions for desired quantities are obtained. This greatly reduces the conservatism introduced by interval methods and may, in fact, be the "right" way to introduce interval uncertainty into the models of interest. 
As an alternative to interval methods, these discussers propose the use of uniforr probability distributions. We have explored the relationship of uniform distributions and intervals in [C2]. Problems associated with nonlinearities become difficult to handle when using uniform distributions: distributions quickly become nonuniform. In fact, they nften (but not always) become approximately gaussian. A better approach may be to either work with general distributions all along, or to come up with procedures for approximately converting intervals into distributions a-posteriori. All these topics required further exploration.

In response to the comments by Dr. Miranda, one can say that either interval methods are a special case of fuzzy set methods, or that fuzzy set methods are a generalization of interval methods, depending on the perspective one wishes to have. As pointed out by the discusser and by most references on fuzzy arithmetic, regardless of the point of view interval methods are usually an essential part of the fuzzy arithmetic computational process: at any desired $\alpha$-cut level one deals with intervals [C2,C3]. And, in spite of the fuzzy nature of a problem characterization, one must assume a specific possibilistic distribution before computations can proceed.

The other point made by Dr. Miranda is that comparisons with stochastic models should not be made. We agree that there is a fundamental difference between possibilistic approaches and probabilistic ones. We also agree that both approaches are cooperative rather than competing, and that the type of information that one gets is very different. We do not agree, however, that a parallel should not be attempted. In fact, a properly done comparison of the two approaches can be very enlightening [C2]. In some cases the probabilistic point of view is more appropriate, while in others the interval and/or possibilistic (fuzzy) point of view leads to a more natural interpretation of results.

[C1] F. Alvarado, Y. Hu, D. Ray, R, Stevenson, E. Cashman "Engineering Foundations for the Determination of Security Costs," IEEE Transactions on Power Systems, August 1991, Vol. 6, No. 3, pp. 1175-1182.

[C2] F. L. Alvarado, "Capturing Uncertainty in Power System Models and Computations," Norman. Oklahoma, July 15-17, 1991 (invited panel presentation).

[C3] A. Kaufmann and M. M. Gupta, "Introduction to Fuzzy Arithmetic," Van Nosirand Reinhold Co., 1985.

Manuscript received December 2, 1991. 